\title{
ARTICLE
}

\section{Measurement of radiation environment inside residential houses in radioactive contaminated areas due to the Fukushima nuclear accident}

\author{
Masashi Takada ${ }^{a^{*}}$, So Kamada, Kazuaki Yajima, Kazuki Iwaoka, Hiroko Enomoto, Hiroyuki Tabe, Hidenori Yonehara \\ and Nobuyuki Sugiura \\ ${ }^{a}$ National Institute of Radiological Sciences, 3-9-1 Anagawa, Inage-ku, Chiba, 263-8555, Japan
}

\begin{abstract}
The radiation environment inside residential houses was measured at MinamiSoma-shi and Iitate-mura in Fukushima Prefecture. The average measured reduction coefficients, which are the ratios of the indoor and outdoor ambient-dose-equivalent rates were 0.35 and 0.45 at the centers of the first and second floors, respectively. The indoor dose rates on the second floors were larger than those on the first floors. The indoor dose rates increased with measurement height. The indoor and outdoor photon energy spectra were measured, revealing that the indoor scattered photon component is larger than the outdoor one.
\end{abstract}

Keywords: Photon; Fukushima nuclear accident; Reduction coefficient; Ambient-dose-equivalent rate; Energy spectrum; Residential house interior

\section{Introduction}

On March 11, 2011, devastating earthquakes off the coast of Japan generated a huge tsunami, which swept over the Fukushima-Daiichi Nuclear Power Station. Devastatingly, the destructive tsunami subsequently disabled the emergency generators required to cool the reactors. There was evidence of partial nuclear meltdowns in units 1,2 , and 3. A massive amount of radioactivity was released from the nuclear facility, which was diffused by wind into the environment over a large area of Japan.

Ten months after the accident, the ground was still contaminated by radioactivity in a wide area of Fukushima Prefecture. Residents in this area have also been exposed to radiation. During the year following the earthquake, many radiation environment monitors, which were installed in a wide area of Fukushima Prefecture to observe the radiation level, measure only the outdoor ambient-dose-equivalent rates. Residents spend more time in houses, offices and schools than in outdoor activities at work and school and playing outdoors. The outdoor activities add up to approximately 5-6 h per day and at most 8 h. For the rest of time, approximately 18-19 h per day, residents stay indoors for activities such as sleeping, working, studying, and taking meals. The indoor radiation environment differs from outdoors. The exposure doses of residents are evaluated using the reduction coefficient for radiation levels in houses and buildings.

The International Atomic Energy Agency (IAEA)

*Corresponding author. Email: m_takada@nirs.go.jp provides the reduction coefficient, which is the ratio of the indoor and outdoor ambient-dose-equivalent rates, for evaluating indoor exposure doses [1]. The outdoor dose rates are measured, and the indoor dose rate can be obtained by multiplying the outdoor dose rate with the reduction coefficient. The resident exposure dose rate is evaluated using the outdoor and indoor dose rates and the number of hours of outdoor activity. The provided reduction coefficient is 0.4 with a range of 0.2-0.5 for wooden houses; for concrete and brick houses, the coefficient is 0.2 with a range of $0.04-0.4$. These values are evaluated on the basis of European house style and radioactive contamination. To estimate the residential dose due to the Fukushima nuclear accident, it is necessary to study the radiation environment and reduction coefficient in Japanese houses.

In this study, we describe the reduction coefficient and the indoor radiation environment and photon energy spectra measured in MinamiSoma-shi and Iitate-mura in Fukushima Prefecture in January and March 2012.

\section{Measurements}

\subsection{Measurement area}

The measurements were completed by volunteers from the Soma regional fire department, who provided an opportunity to survey the radiation environment indoors and around their houses. The measurements were obtained at 19 residential houses and 2 fire department buildings in MinamiSoma-shi and Iitate-mura on January 15-16 and March 18, 2012, one year after the Fukushima nuclear accident.

On these dates, the measured environmental dose 
rates were approximately $0.4 \mu \mathrm{Sv} / \mathrm{h}$ at MinamiSoma-shi and $1.5 \mu \mathrm{Sv} / \mathrm{h}$ at Iitate-mura. The natural background in MinamiSoma-shi was $0.06 \mu \mathrm{Sv} / \mathrm{h}$ on the basis of the previous radiation environment in MinamiSoma-shi before the nuclear accident. In these areas, several parks and school yards underwent radioactive decontamination; however, residential gardens and houses have not yet been decontaminated.

The indoor radiation levels were measured at a height of $1 \mathrm{~m}$ at the center of each house, several locations of each room on the first and second floors, and in toilets and bath rooms. The dose distributions with height from the ground were also measured in several houses.

\subsection{Detectors}

The ambient-dose-equivalent rates were measured using $\mathrm{NaI}(\mathrm{Tl})$ scintillation survey meters (TCS-161 and -171, Hitachi Aloka Medical Ltd., Mitaka, Tokyo, Japan). The survey meter consists of an $\mathrm{NaI}(\mathrm{Tl})$ scintillator having diameter and length both of $25.4 \mathrm{~mm}$ and exhibit a flat energy response from $50 \mathrm{keV}$ to 3 $\mathrm{MeV}$. The survey meters were calibrated with a ${ }^{137} \mathrm{Cs}$ photon source at the photon calibration facility in the National Institute of Radiological Sciences, Chiba, Japan. The absorbed dose rates from this photon source were measured using a calibrated $10-\mathrm{L}$ ion chamber (TN-32003, PTW-Freiburg GmbH, Germany) at the National Metrology Institute of Japan in the National Institute of Advanced Industrial Science and Technology, Tsukuba, Japan.

Indoor and outdoor photon energy spectra were measured using $\mathrm{NaI}(\mathrm{Tl})$ scintillator spectrometers (Inspector1000, Canberra Industrial Inc., USA and EMF211, EMF Japan Co., Ltd., Japan) with $76.2 \mathrm{~mm}$ in length and $76.2 \mathrm{~mm}$ in diameter. These spectrometers consist of an $\mathrm{NaI}(\mathrm{Tl})$ scintillator, a high voltage, an amplifier, and a multichannel analyzer. The photon energies at the pulse heights were calibrated using several photopeaks of ${ }^{134,137} \mathrm{Cs}$ and the natural background of ${ }^{40} \mathrm{~K}$ and ${ }^{208} \mathrm{Tl}$ in the measurement. The scintillators were placed inside the houses and around their exteriors at a height of $1 \mathrm{~m}$ above the ground and directed toward the zenith.

Radioactive contamination on the walls and roofing tails of houses was measured using Geiger-Müller survey meters (TGS-146B, Hitachi Aloka Medical Ltd.). The measured radioactive contamination was negligible on the wall. No radioactive contamination was detected on the roofing tails in most of houses; however, in a few houses, radioactivity was detected on the roofing tails as spots exhibiting a few hundred counts per minute. This roofing tail contamination did not affect the radiation level in the house.

Radionuclides in the outdoor radiation environments were identified using a portable high-purity Ge detector (Micro Detective, ORTEC, AMETEK Inc., USA) and $\mathrm{LaBr}(\mathrm{Ce})$ scintillator (Inspector1000, Canberra Ind. Inc.). These detectors were used on the ground to measure photons from the soil. The following radionuclides were separated from each other with high energy resolution: ${ }^{134,137} \mathrm{Cs}$ and ${ }^{110 \mathrm{~m}} \mathrm{Ag}$ as artificial radionuclides and ${ }^{40} \mathrm{~K}$, ${ }^{214} \mathrm{Bi},{ }^{208} \mathrm{Tl}$ and ${ }^{228} \mathrm{Ac}$ as natural radionuclides. On the measurement days, the fraction of radioactivity was $45 \%$ for ${ }^{134} \mathrm{Cs}$, $55 \%$ for ${ }^{137} \mathrm{Cs}$, and $0.0015 \%$ for ${ }^{110 \mathrm{~m}} \mathrm{Ag}$. Soil samples from around the houses were collected to measure the radioactivity in detail. The same ratios of artificial radionuclides were obtained in a laboratory.

\section{Results and discussion}

\subsection{Indoor radiation environment}

The ambient-dose-equivalent rates were measured at the centers of the houses and at the several locations in the rooms on the first and second floors using the $\mathrm{NaI}(\mathrm{Tl})$ survey meters. The dose rates depended on the location in the houses with the lowest dose rate appearing at the center of the first floor. The following are the dose rates from lowest to highest: (1) at the center of the first floor, (2) at the center of the second floor, (3) other locations on the first floor, and (4) other locations on the second floor.

The indoor dose rate is plotted versus the outdoor dose rate in Figure 1. The natural background dose rate of $0.06 \mu \mathrm{Sv} / \mathrm{h}$ was subtracted from the observed outdoor dose rates. The obtained natural background for the indoor dose rates is $0.048 \mu \mathrm{Sv} / \mathrm{h}$, including a provisional reduction coefficient of 0.8 , obtained from the photon energy spectra.

The indoor dose rates at the centers of the houses and in other locations within the houses are plotted in Figure 1-(A) and (B), respectively. Those on the first and second floors are indicated by black and red closed circles, respectively. Medium, upper, and lower values of the reduction coefficients, provided by the IAEA for the center of wooden houses, are drawn as black dashed lines with labels. The dose rates on the first and second floors are fitted as linear correlations, which are drawn as black and red solid lines, respectively.

\subsection{Reduction coefficient}

The average reduction coefficients were obtained by fitting the distributions of the ratios of the indoor and outdoor dose rates with Gaussian distributions. The distributions were separately analyzed at four locations: the centers of the first and second floors and the other areas of first and second floors. Reduction coefficients of $0.35,0.45,0.48$, and 0.55 , respectively, were obtained, as listed in Table 1. From the reduction coefficients at the centers of both the first and second floors, the average reduction coefficient at the centers of the houses is 0.4 with a standard deviation of 0.18 . This coefficient agrees well with that provided by the IAEA, although the dose-rate measurements were performed in European houses. The comparison with the reduction coefficient provided by the IAEA indicates that the calculation of the exposure doses of residents in wooden houses is valid.

The reduction coefficients in areas other than the 
centers of houses have never been provided by the IAEA. These reduction coefficients, obtained at the several locations in each room and in toilets and bathrooms, are larger than those at the centers of the houses. The dose ratios depend on the window sizes. In toilets and bathrooms, the dose ratios are small; however, in living rooms with large windows, the dose ratios are large because of the small shielding against photons entering the rooms. Walls are effective as photon shields. To reduce the exposure doses, residents should spend more time at the center of the first floor and in rooms with small windows.

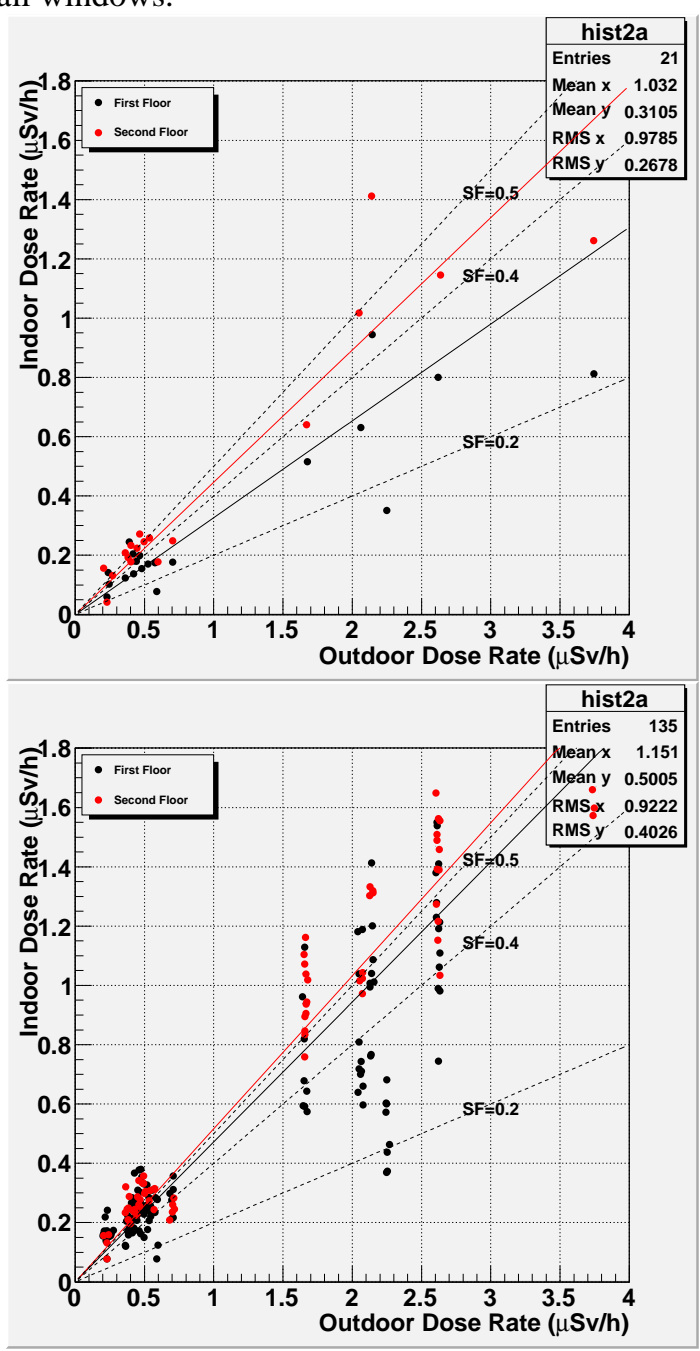

Figure 1. Indoor and outdoor ambient-dose-equivalent rates in (A) center and (B) other areas on the first floors (black closed circles) and second floors (red closed circles). Solid lines fit the dose rates as a linear correlation, and dashed lines are the reduction coefficients provided by the IAEA and labeled with the values [1].

Table 1. Reduction coefficients.

\begin{tabular}{ccccc}
\hline \multirow{2}{*}{ Location } & \multicolumn{2}{c}{ Center } & \multicolumn{2}{c}{ Other than center } \\
\cline { 2 - 5 } & $1^{\text {st }} \mathrm{FL}$ & $2^{\text {nd }} \mathrm{FL}$ & $1^{\text {st }} \mathrm{FL}$ & $2^{\text {nd }} \mathrm{FL}$ \\
\hline Reduction Coeff. & 0.35 & 0.45 & 0.48 & 0.55 \\
Standard dev. & 0.13 & 0.12 & 0.15 & 0.10 \\
\hline
\end{tabular}

The indoor and outdoor ambient-dose-equivalent rates were measured from June 2011 to June 2012 at the Iitate fire department, which is a single-story concrete building. The average reduction coefficient at the center of the building was 0.28 with a standard deviation of 0.06. Even after the radioactive contaminated soil around the building was cleaned several times, the reduction coefficient is remained. The IAEA provides a reduction coefficient of 0.2 with a range of 0.04-0.4 for concrete buildings. The authors' coefficient is larger than that provided by the IAEA but within its range. This large value is attributed to the large window in the room. The radiation environment in the concrete building should be studied further.

The indoor and outdoor ambient-dose-equivalent rates were measured at a height of $1 \mathrm{~m}$ above the floor level in the houses and above the ground, respectively. In addition, the indoor and outdoor dose rates in several houses were measured at several heights, plotted as solid lines with closed circles and dashed lines with opened circles in Figure 2, to observe the height dependence of the dose rates. The heights in the houses are defined from the ground, including the floor height and roof thickness on the basis of the dimensions of a typical house. The first and second floors cover heights from 0.5 to $3 \mathrm{~m}$ and from 3.2 to $6 \mathrm{~m}$, respectively, in the houses. The outside dose rates decreased as the measurement height increased, whereas the indoor dose rates increased as the height increased on both the first and second floors. The indoor dose-rate curves with height differ from the outdoor ones. This difference is attributed to several shields around the houses and wider solid angles at higher positions. This result will be studied further.

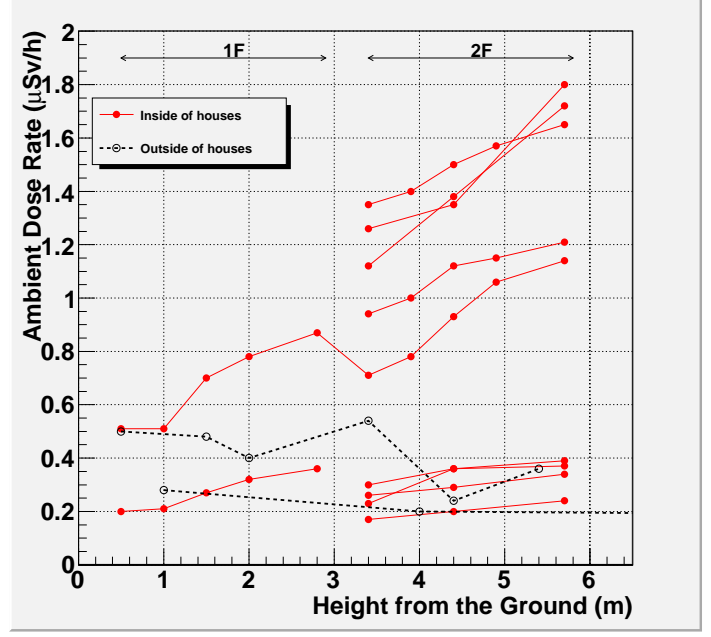

Figure 2. Indoor and outdoor ambient-dose-equivalent rates as a function of height (solid lines with closed circles and dashed lines with open circles, respectively).

\subsection{Photon energy spectra}

\subsubsection{Photon energy spectra}

The indoor and outdoor pulse height distributions were measured using the $\mathrm{NaI}(\mathrm{Tl})$ scintillation 
spectrometers. Several photopeaks of the artificial radionuclides, ${ }^{134,137} \mathrm{Cs}$, and the natural background, ${ }^{40} \mathrm{~K}$ and ${ }^{208} \mathrm{Tl}$ were observed in the pulse heights. The other radionuclides were not identified. The scattered components of ${ }^{134,137} \mathrm{Cs}$ in the atmosphere and on the ground were measured; the indoor scattered component is larger than the outdoor one.

The indoor and outdoor photon energy spectra at Iitate-mura were obtained by unfolding the pulse height distributions with the FerdoU code; they are plotted as solid and dashed lines, respectively, in Figure 3. The photon energy spectra range from 0 to $3 \mathrm{MeV}$, including natural radionuclides. They are normalized by the measurement times. The outdoor photon energy spectra were measured at the northeast and northwest positions around the house (black and red solid lines, respectively); the indoor photon spectra were measured at the centers of the first and second floors (black and red dashed lines, respectively). Photon peaks for the radionuclides ${ }^{134,137} \mathrm{Cs},{ }^{40} \mathrm{~K}$, and ${ }^{208} \mathrm{Tl}$ are indicated by arrows. The photon energy spectra mainly consist of three components: photons directly emitted from ${ }^{134,137}$ Cs radionuclides from 0.5 to $1.4 \mathrm{MeV}$, photons scattered in the atmosphere and the ground from 0 to 0.5 $\mathrm{MeV}$, and the natural background from 1.4 to $3 \mathrm{MeV}$. The indoor photon energy spectra are $70 \%$ smaller than those of the outdoor ones; both the energy spectra show similar distributions, but the scatter components differ. The ratios of scattered to direct photon fluxes inside and outside the houses are 1.80 and 1.05 , respectively.

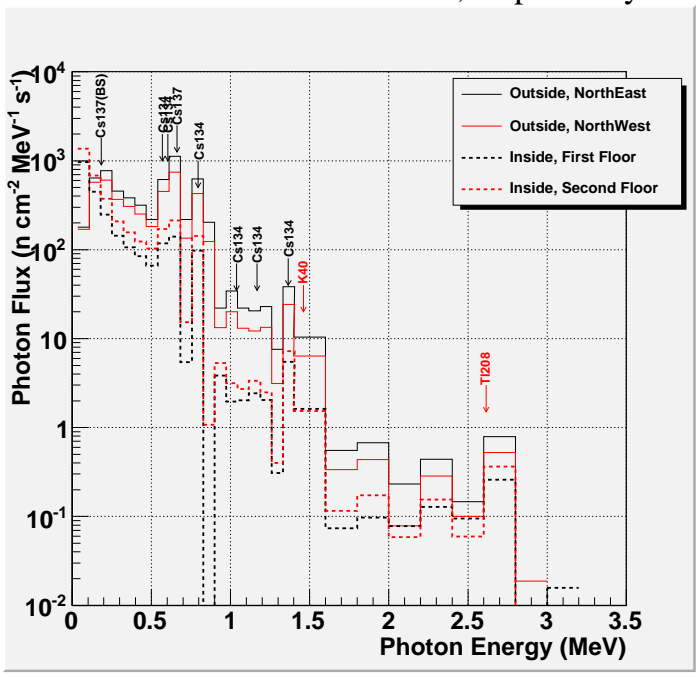

Figure 3. Indoor and outdoor energy spectra at Iitate-mura. Outdoor energy spectra were measured at northeast and northwest positions around the house; indoor energy spectra were measured at the centers of the first and second floors.

\subsubsection{Reduction coefficient}

From the direct photon components observed from 0.5 to $0.8 \mathrm{MeV}$, the obtained reduction coefficient of only direct photons is 0.29 . This coefficient is $30 \%$ smaller than those measured using the $\mathrm{NaI}(\mathrm{Tl})$ survey meters because of the absence of the scattered and natural background photon components. When the scattered and natural background photons are included, the reduction coefficient obtained from the indoor and outdoor photon energy spectra becomes 0.37 with a standard deviation of 0.13 . The average reduction coefficient evaluated from the photon energy spectra agrees with those measured using the survey meters (0.40). This agreement indicates that the evaluation using the survey meters with the flat energy response was valid.

Several dose ratios inside and outside the houses were evaluated from the photon energy spectra using the ICRP-74 conversion coefficients [2]. The evaluated ratio of the ambient to the absorbed dose rates in air is 1.23. The ratios of the effective dose rates for three exposure geometries, isotropic, rotation, and anterior posterior to the ambient dose rates are 0.68, 0.83, and 1.08, respectively. These values are useful for estimating the exposure doses of members of the public.

\section{Conclusion}

The indoor and outdoor ambient-dose-equivalent rates were measured at several residential houses in MinamiSoma-shi and Iitate-mura, Fukushima Prefecture. The average reduction coefficients obtained were 0.35 and 0.45 at the centers of the first and second floors, respectively, in wooden houses and 0.28 for the single-story concrete building. The mean of the coefficients at the centers of the houses (0.4) agrees well with the coefficient provided by the IAEA for wooden houses. The indoor dose rates differ with the location. The dose rates at the center of the second floor are larger than those of the first floor. The dose rates at areas other than the centers of the houses are larger than those at the center. Overall, the rate at the center of the first floor the dose rate is lowest. In addition, the indoor dose rates increased with measurement height.

The indoor and outdoor photon energy spectra consist of three components: direct photons from the artificial radionuclides of ${ }^{134,137} \mathrm{Cs}$, photons scattered from the atmosphere and ground, and the natural background. The indoor scattered components (1.80) are larger than those of the outdoor ones (1.05). These reduction coefficients and photon energy spectra are useful for reducing the exposure doses of residents.

\section{Acknowledgements}

The authors' data were gathered by volunteers from the Soma regional fire department. The authors offer their heartfelt appreciation to the Soma regional fire department.

\section{References}

[1] International atomic energy agency, Generic Procedures for Monitoring in a Nuclear or Radiological Emergency, Radiation Safety Section, International Atomic Energy Agency, Austria: IAEA-TECDOC-1162, (2000).

[2] International Commission on Radiological Protection, Conversion Coefficients for Use in Radiological Protection Against External Radiation, Publication 74, Pergamon Press, (1995). 